\title{
On Singular Perturbations of Flexible and Variable-Speed Wind Turbines
}

\author{
R. Oulad Ben Zarouala, ${ }^{1,2}$ C. Vivas, ${ }^{1}$ J. Á. Acosta, ${ }^{1}$ and L. El Bakkali ${ }^{2}$ \\ ${ }^{1}$ Departamento de Ingeniería de Sistemas y Automática, Universidad de Sevilla, Camino de los Descubrimientos $s / n$, \\ 41092 Sevilla, Spain \\ ${ }^{2}$ Team Modelling and Simulation of Mechanical Systems, Faculty of Sciences at Tetouan, University Abdelmalek Essaadi, \\ BP 2121, M'Hannech, 93002 Tetouan, Morocco
}

Correspondence should be addressed to J. Á. Acosta, jaar@esi.us.es

Received 31 October 2011; Accepted 5 February 2012

Academic Editor: Paul Williams

Copyright ( $) 2012$ R. Oulad Ben Zarouala et al. This is an open access article distributed under the Creative Commons Attribution License, which permits unrestricted use, distribution, and reproduction in any medium, provided the original work is properly cited.

\begin{abstract}
A model for the mechanical dynamics of a wind turbine is developed, which is the composition of three physical mechanisms: flexion, torsion, and rotational dynamics. A first contribution is the identification of the essential physical parameters that provide a time-scale separation of these three mechanisms. Under the assumption of singular perturbations the time-scale separation allows to work with a reduced model of order one. This reduction has been essential for the control of this system allowing to control designers to take into account only the reduced-order model. A second contribution consists in employing a measurement of the fore-aft nacelle acceleration with the reduced model, together with a Kalman filter to estimate the flexible DOFs of the system (tower and average blade deflection). The successful approach is tested on high-order nonlinear aeroelastic simulator (FAST).
\end{abstract}

\section{Introduction}

In the past few years, variable-speed wind turbines research and design has become increasingly concerned with control system design. This concern arises for several reasons: turbines have become larger, control system hardware has become more powerful, control is a way to drive down costs and increase performance, and turbine modeling tools have become more sophisticated. As wind turbines increased in size and power, control specifications became more demanding and regulation mechanisms more sophisticated. Increasingly, control systems have been expected not merely to keep the turbine within its safe operating region but also to improve efficiency and quality of power conversion. They have gradually evolved in consequence until playing today an important role in modern energy generation systems [1].

The design of the controller must take into account the effect on loads and at least ensure that excessive loads will not result from the control action [2]. Many wind turbines control systems are based on linear models (see, e.g., [3-5]). This is due to several reasons, as the possibility to obtain simple analytical solutions to many control problems or the simpler implementation of such controllers in practical applications. Most of works describe the wind turbine dynamics as that of the drive train model, neglecting flexible structural modes. Generally, the drive-train consists of a lowspeed shaft, gearbox, high-speed shaft and generator. For example, in $[6,7]$ a one-mass model of drive train is used to describe the wind turbine dynamics. This model cannot account for the flexibility of the low-speed shaft. However, this flexibility induces flexible resonant and nonresonant modes that can cause system oscillations [2]. In the literature, a two-mass model of the drive train is commonly used to describe the wind turbine dynamics (see, e.g., $[2,8-10]$ ). The control law deduced from this model is more general and can be applied for wind turbines of different sizes. Particularly, these controllers are better adapted for high-flexibility wind turbines that cannot be properly modeled with a one massmodel [7].

Much progress has been made in recent years in the developing of complex yet accurate aeroelastic simulators for wind turbines, providing good predictions of loads 

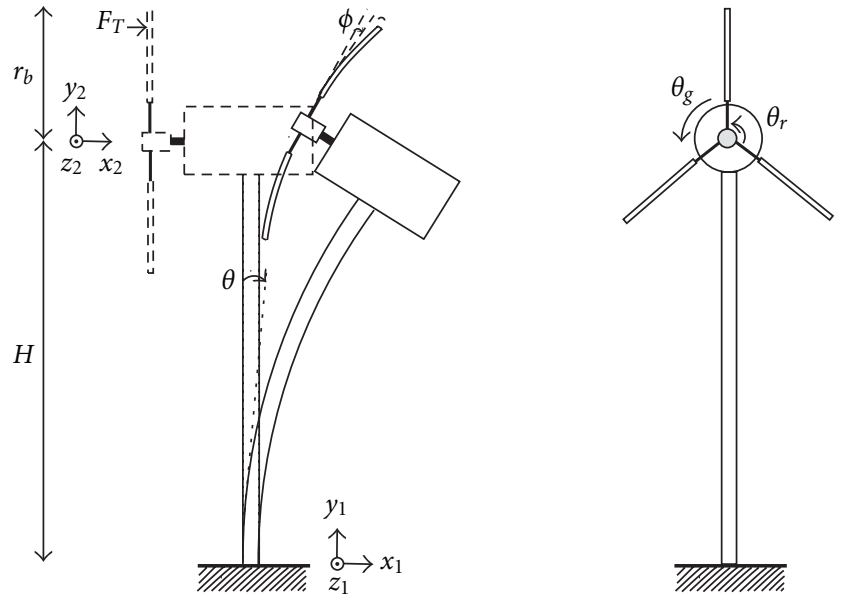

FIGURE 1: Mode shapes for horizontal-axis wind turbines.

and performance. However, the complexity of these models cannot be handled for control purposes and lower-order linearized models are usually preferred. Obtaining low-order accurate system models for wind turbine control design has proven to be difficult because of the particular operating conditions. Moreover, the current tendency towards larger and more flexible wind turbines is making this task even more involved. The contributions of this work are twofold. On the one hand a nonlinear model of moderate order is developed, taking into account drive train, tower, and blades flexibilities. From this model a linearised model is derived, which is analysed in the light of the theory of singular perturbation theory. With this approach the work clearly identifies three physical subsystems whose main dynamics can be separated in different time scales, namely, flexion, torsion, and rotational dynamics. The first contribution of this work is the identification of the essential physical parameters which provide a time-scale separation of these three mechanisms, providing conditions for this separation to hold.

The second contribution of this work consists in proposing a Kalman filtering structure to estimate the flexible DOF of tower and blades. In order for this filter to work, it is proposed to postulate the installation of an accelerometer on the nacelle, so that its fore-aft acceleration can be measured and fed to the filter.

It is shown how this additional data can be employed together with the reduced flexion subsystem to successfully estimate tower and blade deflections. The approach is tested by simulation on high-order nonlinear aeroelastic simulator (FAST), showing good results in recovering essential information for load damage evaluation.

\section{Model of the Wind Turbine}

In this section a mechanical model of WECS is developed including deformations due to the flexibility of the blades and the tower. To this end, in Figure 1 two reference systems have been defined, one attached to the base of the tower
TABLE 1: Parameters of the WECS mode.

\begin{tabular}{ll}
\hline Symbol & Description \\
\hline$m_{t}$ & Mass of the tower \\
$m_{p}$ & Mass of each blade \\
$I_{t}$ & Inertia of the tower \\
$I_{p}$ & Inertia of blades \\
$J_{r}$ & Inertia of the rotor \\
$J_{g}$ & Generator inertia (low-speed side) \\
$k_{t}$ & Stiffness of the tower \\
$k_{p}$ & Stiffness of each blade \\
$k_{s}$ & Stiffness of the transmission \\
$N$ & Number of blades \\
$R$ & Length of each blade \\
$H$ & Height of the tower \\
$a$ & Length of the nacelle \\
$B_{t}$ & Damping of the tower \\
$B_{p}$ & Damping of each blade \\
$B_{s}$ & Damping of the transmission \\
$B_{r}$ & Rotor external damping \\
$B_{g}$ & Generator external damping \\
$n_{g}$ & Gearbox Ratio \\
$H_{g}$ & Distance to the mass center of the tower \\
$R_{g}$ & Distance to the mass center of the blade \\
\hline &
\end{tabular}

$S_{1}:\left(O_{1} ; x_{1}, y_{1}, z_{1}\right)$ and another attached to the bladeand-shaft joint $S_{2}:\left(O_{2} ; x_{2}, y_{2}, z_{2}\right)$. The set of generalised coordinates of the model of the wind turbine is defined as $q=\left[\theta, \phi, \theta_{r}, \theta_{g}\right]^{T} \in S^{4}$, where $\theta$ is the angular position of the tower, $\phi$ is the angular position of the blade out of the plane of rotation, $\theta_{r}$ is the angular position of the rotor, and $\theta_{g}$ is the angular position of the generator. Let $\Theta_{r}^{i}$ be defined as

$$
\Theta_{r}^{i}:=\theta_{r}+i \frac{2 \pi}{N}, \quad i=0,1, \ldots, N-1,
$$

where $N$ is the total number of blades. Thus, The position of a point $Q$ at a distance $r$ of the reference system $S_{2}$ is given by

$$
\left.Q\right|_{S_{2}}:\left\{\begin{array}{l}
x=-r \sin \phi, \\
y=r \cos \phi \sin \Theta_{r}^{i}, \\
z=r \cos \phi \cos \Theta_{r}^{i} .
\end{array}\right.
$$

Thus, the componentwise velocity vector of $Q$ with respect to $S_{1}$ can be computed through the well-known formula

$$
\overrightarrow{v / s_{1}}(Q)=\overrightarrow{v / s_{2}}(Q)+\overrightarrow{v / s_{1}}\left(O_{2}\right)+\vec{\Omega}_{S_{2} / S_{1}} \wedge \overrightarrow{O_{2} Q},
$$

with $\Omega$ being the angular velocity of $S_{2}$ with respect to $S_{1}$. Now, we are in a position to derive the mechanical (subsystem) model of a WECS. All the necessary parameters of the model are defined in Table 1.

Using Lagrange's equations [11], the dynamic windturbine model with $N$ blades is given by

$$
\frac{d}{d t}\left(\nabla_{\dot{q}} E_{k}\right)+\nabla_{\dot{q}} E_{d}-\nabla_{q} E_{k}+\nabla_{q} E_{p}=G u,
$$


where $E_{k}, E_{d}$, and $E_{p}$ denote the kinetic, dissipative, and potential energies, respectively, and $G$ the input control matrix with the control input defined as $u=\left[\begin{array}{lll}F_{T} & T_{r} & T_{g}\end{array}\right]^{T} \in$ $\mathbb{R}^{3}$ with $T_{g}$ and $T_{r}$ being the generator and aerodynamic torques, respectively, and the thrust forces distributed along each blade were replaced by a lumped force $F_{T}$ applied at a distance $r_{b}$ from the axis of rotation. The kinetic $\left(E_{k}\right)$ and dissipative $\left(E_{d}\right)$ energies are given, respectively, by

$$
\begin{aligned}
E_{k}= & \frac{N}{2} m_{p}\left[\frac{R^{2}}{3} \dot{\phi}^{2}+R H \cos \phi \dot{\phi} \dot{\theta}+\frac{R^{2}}{3} \cos ^{2} \phi \dot{\theta}_{r}^{2}\right. \\
& \left.+\left(H^{2}+a^{2}+R a \sin \phi+\frac{R^{2}}{6}\left(1+\sin ^{2} \phi\right)\right) \dot{\theta}^{2}\right] \\
& +\frac{I_{t}}{2} \dot{\theta}^{2}+\frac{J_{r}}{2} \dot{\theta}_{r}^{2}+\frac{J_{g}}{2} \dot{\theta}_{g}^{2}, \\
E_{d}= & \frac{B_{t}}{2} \dot{\theta}^{2}+\frac{N B_{p}}{2} \dot{\phi}^{2}+\frac{B_{s}}{2}\left(\dot{\theta}_{r}-\frac{\dot{\theta}_{g}}{n_{g}}\right)^{2}+\frac{B_{r}}{2} \dot{\theta}_{r}^{2}+\frac{B_{g}}{2} \dot{\theta}_{g}^{2},
\end{aligned}
$$

while the potential energy $\left(E_{p}\right)$ can be decomposed as

$$
E_{p}=E_{g}+E_{f}+E_{t}
$$

with

$$
\begin{aligned}
E_{g}= & N m_{p} g(H(1-\cos \theta)-a \sin \theta) \\
& +m_{t} g H_{g}(1-\cos \theta), \\
E_{f}= & \frac{k_{t}}{2} \theta^{2}+N \frac{k_{p}}{2} \phi^{2}, \\
E_{t}= & \frac{k_{s}}{2}\left(\theta_{r}-\frac{\theta_{g}}{n_{g}}\right)^{2},
\end{aligned}
$$

where $E_{g}$ is due to the gravity action, $E_{f}$ is due to the flexibility of the blades and the tower, and $E_{t}$ is due to the torsion along the shaft referred to the low-speed side. Let $\theta_{s}=\theta_{r}-\theta_{g} / n_{g}$ be the torsion angle. Thus, redefining a new set of generalised coordinates as $q=\left[\theta, \phi, \theta_{s}, \theta_{g}\right]^{T}$ and linearising Lagrange's equation (4), the equation of motion becomes

$$
M \ddot{\bar{q}}+\bar{C} \dot{\bar{q}}+K \bar{q}=G \bar{u},
$$

where for compactness we defined $\bar{C}=C+B$. Let $q_{\mathrm{eq}}, u_{\mathrm{eq}}$ be the coordinates and torques evaluated at the operation point; then the vectors $\bar{q}$ and $\bar{u}$ represent deviations from that operating point, as $\bar{q}=q-q_{\mathrm{eq}}$ and $\bar{u}=u-u_{\mathrm{eq}}$; $M\left(q_{\mathrm{eq}}\right) \in \mathbb{R}^{4 \times 4}$ the inertia matrix, $C\left(q_{\mathrm{eq}}, \dot{q}_{\mathrm{eq}}\right) \in \mathbb{R}^{4 \times 4}$ the Coriolis and gyroscopic torques, $B \in \mathbb{R}^{4 \times 4}$ the damping torques, $K\left(q_{\mathrm{eq}}\right)$ the stiffness matrix, and $G_{\mathrm{eq}}\left(q_{\mathrm{eq}}\right) \in \mathbb{R}^{4 \times 3}$ the control matrix. These matrices are given as follows:

$$
M\left(\phi_{\mathrm{eq}}\right)=\left[\begin{array}{cccc}
m_{1} & m_{2} & 0 & 0 \\
m_{2} & m_{3} & 0 & 0 \\
0 & 0 & m_{4} & \frac{m_{4}}{n_{g}} \\
0 & 0 & 0 & m_{5}
\end{array}\right],
$$

where

$$
\begin{aligned}
m_{1}= & I_{t}+N m_{p}\left(H^{2}+a^{2}\right) \\
& +N m_{p} R a \sin \phi_{\mathrm{eq}} \\
& +\frac{I_{p}}{2}\left(1+\sin ^{2} \phi_{\mathrm{eq}}\right), \\
m_{2}= & \frac{N}{2} m_{p} R H \cos \phi_{\mathrm{eq}}, \\
m_{3}= & I_{p}, \\
m_{4}= & J_{r}+I_{p} \cos ^{2} \phi_{\mathrm{eq}}, \\
m_{5}= & \left.J_{g}, \quad \begin{array}{cccc}
0 & 0 & 0 & 0 \\
0 & 0 & C_{23} & \frac{C_{23}}{n_{g}} \\
0 & -C_{23} & 0 & 0 \\
0 & 0 & 0 & 0
\end{array}\right] \\
\bar{C}\left(\phi, \theta_{s}, \theta_{g}\right)_{\mathrm{eq}}= & \\
& +\left[\begin{array}{cccc}
B_{t} & 0 & 0 \\
0 & N B_{p} & 0 \\
0 & 0 & B_{r}+B_{s} \\
0 & 0 & -\frac{B_{s}}{n_{g}} & B_{g}+\frac{B_{s}}{n_{g}}-\frac{B_{s}}{n_{g}^{2}}
\end{array}\right],
\end{aligned}
$$

with

$$
\begin{aligned}
& C_{23}=I_{p} \sin 2 \phi_{\mathrm{eq}}\left(\dot{\theta}_{s_{\mathrm{eq}}}+\frac{\dot{\theta}_{g_{\mathrm{eq}}}}{n_{\mathrm{g}}}\right), \\
& K\left(\theta, \phi, \theta_{s}, \theta_{g}\right)_{\mathrm{eq}}=\left[\begin{array}{cccc}
k_{1} & 0 & 0 & 0 \\
0 & k_{3} & 0 & 0 \\
0 & 0 & k_{s} & 0 \\
0 & 0 & -\frac{k_{s}}{n_{g}} & 0
\end{array}\right],
\end{aligned}
$$

with

$$
\begin{aligned}
k_{1}= & k_{t}-m_{t} g H_{g} \cos \theta_{\mathrm{eq}}-N m_{p} g \\
& \times\left(H \cos \theta_{\mathrm{eq}}+a \sin \theta_{\mathrm{eq}}\right)+N H \sin \theta_{\mathrm{eq}} F_{T_{\mathrm{eq}}}, \\
k_{3}= & I_{p} \cos 2 \phi_{\mathrm{eq}}\left(\dot{\theta}_{s_{\mathrm{eq}}}+\frac{\dot{\theta}_{g_{\mathrm{eq}}}}{n_{g}}\right)^{2} \\
& +N k_{p}+N r_{b} \sin \phi_{\mathrm{eq}} F_{T_{\mathrm{eq}}} .
\end{aligned}
$$

Finally, the control matrix reads

$$
G(\theta, \phi)_{\mathrm{eq}}=\left[\begin{array}{ccc}
N H \cos \theta_{\mathrm{eq}} & 0 & 0 \\
N r_{b} \cos \phi_{\mathrm{eq}} & 0 & 0 \\
0 & 1 & 0 \\
0 & 0 & -1
\end{array}\right] .
$$


The equilibrium or operation point can be computed through the nonlinear algebraic equations given by

$$
\begin{aligned}
& \theta_{\mathrm{eq}}=\frac{1}{k_{t}}\left(m_{t} g H_{g} \sin \theta_{\mathrm{eq}}+N m_{p} g\left(H \sin \theta_{\mathrm{eq}}-a \cos \theta_{\mathrm{eq}}\right)\right. \\
& \left.+N H \cos \theta_{\mathrm{eq}} F_{T_{\mathrm{eq}}}\right) \\
& \phi_{\mathrm{eq}}=\frac{1}{N k_{p}} \\
& \left(N r_{b} \cos \phi_{\mathrm{eq}} F_{T_{\mathrm{eq}}}-\frac{I_{p}}{2} \sin 2 \phi_{\mathrm{eq}}\left(\dot{\theta}_{s_{\mathrm{eq}}}+\frac{\dot{\theta}_{g_{\mathrm{eq}}}}{n_{g}}\right)^{2}\right), \\
& \theta_{s_{\mathrm{eq}}}=\frac{1}{k_{s}}\left(T_{r_{\mathrm{eq}}}-\frac{B_{r}}{n_{g}} \dot{\theta}_{g_{\mathrm{eq}}}-\left(B_{r}+B_{g}\right) \dot{\theta}_{s_{\mathrm{eq}}}\right) \text {, } \\
& T_{g_{\mathrm{eq}}}=\frac{B_{s}}{n_{g}} \dot{\theta}_{s_{\mathrm{eq}}}-B_{g} \dot{\theta}_{g_{\mathrm{eq}}}+\frac{k_{s}}{n_{g}} \theta_{s_{\mathrm{eq}}}, \\
& \dot{\theta}_{\mathrm{eq}}=0 \text {, } \\
& \dot{\phi}_{\mathrm{eq}}=0 \text {, } \\
& \frac{d}{d t}\left[\begin{array}{c}
\bar{\theta} \\
\bar{\phi} \\
\dot{\bar{\theta}} \\
\dot{\bar{\phi}}
\end{array}\right]=\left[\begin{array}{cccc}
0 & 0 & 1 & 0 \\
0 & 0 & 0 & 1 \\
a_{5} & a_{6} & a_{1} & a_{2} \\
b_{5} & b_{6} & b_{1} & b_{2}
\end{array}\right]\left[\begin{array}{c}
\bar{\theta} \\
\bar{\phi} \\
\dot{\bar{\theta}} \\
\dot{\bar{\phi}}
\end{array}\right]+\left[\begin{array}{c}
0 \\
0 \\
a_{7} \\
b_{7}
\end{array}\right] \bar{F}_{T} \\
& +\left[\begin{array}{ll}
0 & 0 \\
0 & 0 \\
0 & a_{3} \\
0 & b_{3}
\end{array}\right]\left[\begin{array}{c}
\bar{\theta}_{s} \\
\dot{\bar{\theta}}_{s}
\end{array}\right]+\left[\begin{array}{c}
0 \\
0 \\
a_{4} \\
b_{4}
\end{array}\right] \dot{\bar{\theta}}_{g} \text {, } \\
& \frac{d}{d t}\left[\begin{array}{c}
\bar{\theta}_{s} \\
\dot{\bar{\theta}}_{s}
\end{array}\right]=\left[\begin{array}{cc}
0 & 1 \\
z_{5} & z_{2}
\end{array}\right]\left[\begin{array}{c}
\bar{\theta}_{s} \\
\dot{\bar{\theta}}_{s}
\end{array}\right]+\left[\begin{array}{c}
0 \\
z_{7}
\end{array}\right] \bar{T}_{g}+\left[\begin{array}{c}
0 \\
z_{6}
\end{array}\right] \bar{T}_{r} \\
& +\left[\begin{array}{cc}
0 & 0 \\
0 & 0 \\
0 & 0 \\
0 & z_{1}
\end{array}\right]^{T}\left[\begin{array}{c}
\bar{\theta} \\
\bar{\phi} \\
\dot{\bar{\theta}} \\
\dot{\bar{\phi}}
\end{array}\right]+\left[\begin{array}{c}
0 \\
z_{3}
\end{array}\right] \dot{\bar{\theta}}_{g} \\
& \frac{d \dot{\bar{\theta}}_{g}}{d t}=d_{2} \dot{\bar{\theta}}_{g}+\left[\begin{array}{ll}
d_{3} & d_{1}
\end{array}\right]\left[\begin{array}{c}
\bar{\theta}_{s} \\
\dot{\bar{\theta}}_{s}
\end{array}\right]+d_{4} \bar{T}_{g} .
\end{aligned}
$$

Moreover, the system (8) can be decomposed as three subsystems as shown in (17)-(19): the subsystem (1) given by (17), corresponding to flexibility of the blades and tower, the subsystem (2) given by (18), corresponding to the torsion of the shaft, and the subsystem (3) given by (19), corresponding to the rotational dynamics of the generator. The corresponding parameters are given in Table 4 .

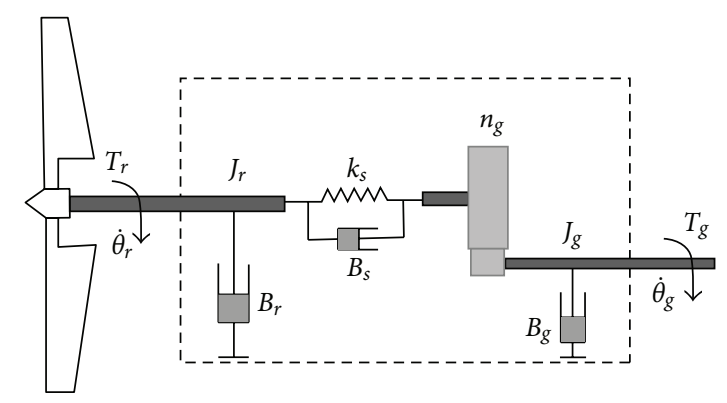

Figure 2: Drive-train.

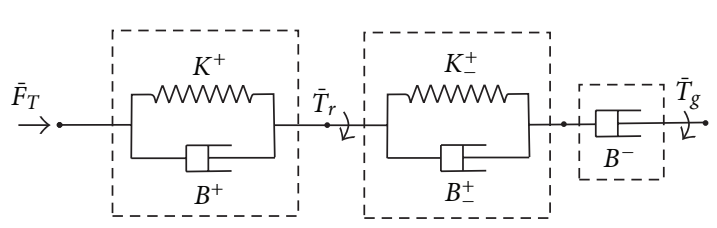

Figure 3: mechanical analogy of the wind turbine.

TABLE 2: Corresponding values of Figure 3.

\begin{tabular}{lcc}
\hline$\star$ & $B \star$ & $K \star$ \\
\hline+ & $\left(\begin{array}{cc}B_{t} & 0 \\
0 & N B_{p}\end{array}\right)$ & $\left(\begin{array}{cc}K_{1} & 0 \\
0 & K_{3}\end{array}\right)$ \\
\pm & $B_{r}+B_{s}$ & $K_{s}$ \\
- & $B_{g}+\frac{B_{s}}{n_{g}}-\frac{B_{s}}{n_{g}^{2}}$ & - \\
\hline
\end{tabular}

Remark 1. The block structure of (17)-(19) suggests a mechanical analogy for the three kinds of forces/torques acting on the wind turbine. Thus, Figures 2 and 3, together with Table 2, shows the corresponding mechanical analogy where different springs and dampers emulate the flexibility, torsion, and friction of the shaft.

\section{The Singularly Perturbed System}

As it was shown above the mechanical dynamics of a wind turbine is a composition of three physical mechanisms: flexibility of the blades and tower, torsion, and rotational dynamics of the shaft. In this section, we prove that these three mechanisms act, actually, in different time scales allowing to work with a reduced model of order of only one. This reduction has been essential for the controller design of wind turbines, since only a reduced model of first order is needed. First, we prove this reduction by selecting the essential physical parameters which provide the decoupling for the reduction. Once these parameters have been identified a reparametrization reveals the singular perturbed structure of the system. To this end, let $\left(Z_{1}, Z_{2}, \dot{\bar{\theta}}_{g}\right) \in \mathbb{R}^{7}$ denote a more compact state vector with $Z_{1}=[\bar{\theta}, \bar{\phi}, \dot{\bar{\theta}}, \dot{\bar{\phi}}]^{T}$ and $Z_{2}=\left[\bar{\theta}_{s}, \dot{\bar{\theta}}_{s}\right]^{T}$. A careful and quite tedious analysis of the physical properties of the system reveals that the parameters 
$\varepsilon_{1}:=-z_{1} /\left(b_{3} n_{g}^{2}\right)>0$ and $\varepsilon_{2}:=d_{1} / z_{3}>0$ allow to transform (17)-(19) into a singular perturbed dynamics as follows:

$$
\begin{gathered}
\varepsilon_{1} \frac{d Z_{1}}{d t}=A_{1} Z_{1}+w_{s_{1}} Z_{2}+w_{g_{1}} \dot{\bar{\theta}}_{g}+w_{f_{1}} \bar{F}_{T}, \\
\varepsilon_{2} \frac{d Z_{2}}{d t}=A_{2} Z_{2}+w_{s_{2}} Z_{1}+w_{g_{2}} \dot{\bar{\theta}}_{g}+w_{r} \bar{T}_{r}+w_{t} \bar{T}_{g}, \\
\frac{d \dot{\bar{\theta}}_{g}}{d t}=d_{2} \dot{\bar{\theta}}_{g}+w_{s_{3}} Z_{2}+d_{4} \bar{T}_{g},
\end{gathered}
$$

where $\varepsilon_{2}>\varepsilon_{1}>0$ and the corresponding elements of the matrices are given in Appendix B. Thus, the fact $\varepsilon_{2}>\varepsilon_{1}$ suggests that the $Z_{1}, Z_{2}$, and $\dot{\bar{\theta}}_{g}$ are the fast, medium, and slow time-scale dynamics, respectively. The latter means, mathematically, that for sufficiently small $\varepsilon_{2}>0$ the timescale separation is satisfied. On the other hand, in (23) and (24) we show the parameters $\varepsilon_{1}$ and $\varepsilon_{2}$ as a function of the physical properties of the system given in Table 1 , and it is not difficult to see that both are dimensionless. The following assumption is now in order. Notice that most of the wind turbines satisfy the assumption by mechanical design:

$$
\begin{gathered}
\varepsilon_{1}=\frac{1}{n_{g}^{2}\left(J_{r}+I_{p} \cos ^{2} \phi_{\mathrm{eq}}\right)}\left(I_{p}-\frac{\left((N / 2) m_{p} R H \cos \phi_{\mathrm{eq}}\right)^{2}}{I_{t}+N m_{p}\left(H^{2}+a^{2}\right)+N m_{p} R a \sin \phi_{\mathrm{eq}}+\left(I_{p} / 2\right)\left(1+\sin ^{2} \phi_{\mathrm{eq}}\right)}\right), \\
\varepsilon_{2}=\frac{B_{s}}{B_{g}+B_{s} / n_{g}-B_{s} / n_{g}^{2}-B_{r} J_{g} /\left(J_{r}+I_{p} \cos ^{2} \phi_{\mathrm{eq}}\right)} .
\end{gathered}
$$

Assumption A. The parameter $\mu$ defined through (23) as $\mu=$ $\varepsilon_{1}\left(J_{r}+I_{p} \cos ^{2} \phi_{\text {eq }}\right)$ is positive.

Some tedious but straightforward calculations show that assumption A is satisfied if the following useful inequalityas a function of some physical properties defined in Table 1holds:

$$
\frac{I_{t}}{I_{p}}>\frac{3}{2}\left(\frac{H}{R}\right)^{2} \Longleftrightarrow \frac{m_{t}}{N m_{p}}>\frac{3}{2} .
$$

The following Proposition states the time-scale separation of the model (20)-(22).

Proposition 2. Consider the parameters given by (23) and (24) and define

$$
\Delta:=\left[B_{g}+\frac{B_{s}}{n_{g}}\left(1-\frac{1}{n_{g}}\right)\right]^{2}-4 \frac{B_{r} J_{g} B_{s}}{\mu},
$$

where $\mu=\varepsilon_{1}\left(J_{r}+I_{p} \cos ^{2} \phi_{e q}\right)$ from (23). Then, the following implications hold:

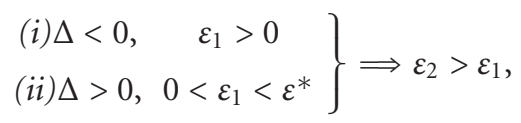

where $\varepsilon^{*}>0$ is defined by

$$
\varepsilon^{*}:=\frac{B_{g}+B_{s} / n_{g}\left(1-1 / n_{g}\right)-\sqrt{\Delta}}{2\left(B_{r} J_{g} / \mu\right)} .
$$

Proof. The inequality $\varepsilon_{1}<\varepsilon_{2}$ is equivalent, from (23) and (24), to

$$
\varepsilon_{1}<\frac{B_{s}}{B_{g}+B_{s} / n_{g}-B_{s} / n_{g}^{2}-B_{r} J_{g}\left(\varepsilon_{1} / \mu\right)},
$$

which in turn is equivalent to $f\left(\varepsilon_{1}\right)>0$, where

$$
f\left(\varepsilon_{1}\right):=\frac{B_{r} J_{g}}{\mu} \varepsilon_{1}^{2}-\left[B_{g}+\frac{B_{s}}{n_{g}}\left(1-\frac{1}{n_{g}}\right)\right] \varepsilon_{1}+B_{s} .
$$

Notice that $f(0)>0$ and $\mu>0$. If $\Delta<0$ then, $f(x)>0$, for any $x \in \mathbb{R}$, and therefore any $\varepsilon_{1}>0$, satisfies $\varepsilon_{2}>\varepsilon_{1}$. If $\Delta>0$ then, $f(x)>0$ for any $x \in\left[0, \varepsilon^{*}\right]$ and therefore $\varepsilon_{1}<\varepsilon^{*}$ satisfies $\varepsilon_{2}>\varepsilon_{1}$.

Table 3 shows the parameters $\varepsilon_{1}$ and $\varepsilon_{2}$ for a number of real wind turbines from [12] and [13].

Remark 3. It should be noticed that $\varepsilon_{1}$ and $\varepsilon_{2}$ are a function of the equilibrium/operation point, and, therefore, the timescale separation should hold for any of them. Thus, to see this in Figure 4 we show $\varepsilon_{1}$ and $\varepsilon_{2}$ as a function of $\phi_{\text {eq }}$ for the WP 1.5 MW wind turbine of Table 3.

3.1. Reduced Dynamics. The theory of singularly perturbed systems is very well known along with the reduction process used in this section $[14,15]$, which underlies the time-scale separation of the system. A controller design for mechanical systems based on singular perturbations is proposed, among others, in [16]. The separation principle relies on the idea that, under some stability assumptions, during the fast transients (so-called boundary-layer), the slow variables remain constant and by the time their changes become noticeable the fast transients have already reached their so-called quasisteady state (equilibrium points with $\varepsilon=0)$. Thus, since the system (20)-(22) has a time-scale separation, then, according to the latter the response of the $Z_{1}$ coordinates of (20) is faster than the response of the $Z_{2}$ coordinates of (21). Roughly speaking, comparing 
TABLe 3: Parameters $\varepsilon_{1}$ and $\varepsilon_{2}$ for real wind turbines.

\begin{tabular}{lcc}
\hline & $\varepsilon_{1}$ & $\varepsilon_{2}$ \\
\hline 5-MW offshore NREL [12] & $1.05 e-004$ & $5.15 e-003$ \\
AWT-27CR2 [13] & $5.33 e-004$ & $2.29 e-002$ \\
AOC-15/50 [13] & $4.18 e-004$ & $1.81 e-002$ \\
WP 1.5 MW [13] & $1.29 e-004$ & $5.68 e-003$ \\
SWRT [13] & $9.83 e-001$ & $5.01 e-001$ \\
\hline
\end{tabular}

Table 4: Parameters for the model (17)-(19).

\begin{tabular}{|c|c|c|c|}
\hline Parameters & Value & Parameters & Value \\
\hline$a_{1}$ & $-\frac{m_{3} \bar{c}_{11}}{l_{1}}$ & $b_{1}$ & $\frac{m_{2} \bar{c}_{11}}{l_{1}}$ \\
\hline$a_{2}$ & $\frac{m_{2} \bar{c}_{22}}{l_{1}}$ & $b_{2}$ & $-\frac{m_{1} \bar{c}_{22}}{l_{1}}$ \\
\hline$a_{3}$ & $\frac{m_{2} \bar{c}_{23}}{l_{1}}$ & $b_{3}$ & $-\frac{m_{1} \bar{c}_{23}}{l_{1}}$ \\
\hline$a_{4}$ & $\frac{m_{2} \bar{c}_{23}}{n_{g} l_{1}}$ & $b_{4}$ & $-\frac{m_{1} \bar{c}_{23}}{n_{g} l_{1}}$ \\
\hline$a_{5}$ & $-\frac{m_{3} k_{1}}{l_{1}}$ & $b_{5}$ & $\frac{m_{2} k_{1}}{l_{1}}$ \\
\hline$a_{6}$ & $\frac{m_{2} k_{3}}{l_{1}}$ & $b_{6}$ & $-\frac{m_{1} k_{3}}{l_{1}}$ \\
\hline$a_{7}$ & $\frac{N\left(m_{3} H \cos \theta_{\mathrm{eq}}-m_{2} r_{b} \cos \phi_{\mathrm{eq}}\right)}{l_{1}}$ & $z_{1}$ & $\frac{\bar{c}_{23}}{m_{4}}$ \\
\hline$b_{7}$ & $-\frac{N\left(m_{2} H \cos \theta_{\mathrm{eq}}-m_{1} r_{b} \cos \phi_{\mathrm{eq}}\right)}{l_{1}}$ & $z_{2}$ & $-\frac{c_{33}}{m_{4}}-\frac{d_{1}}{n_{g}}$ \\
\hline$d_{1}$ & $-\frac{\bar{c}_{43}}{m_{5}}$ & $z_{3}$ & $-\frac{\bar{c}_{34}}{m_{4}}-\frac{d_{2}}{n_{g}}$ \\
\hline$d_{2}$ & $-\frac{\bar{c}_{44}}{m_{5}}$ & $z_{5}$ & $-\frac{d_{3}}{n_{g}}-\frac{k_{s}}{m_{4}}$ \\
\hline$d_{3}$ & $\frac{k_{s}}{m_{5} n_{g}}$ & $z_{6}$ & $\frac{1}{m_{4}}$ \\
\hline$d_{4}$ & $-\frac{1}{m_{5}}$ & $z_{7}$ & $-\frac{d_{4}}{n_{g}}$ \\
\hline$l_{1}$ & $m_{1} m_{3}-m_{2}^{2}$ & $I_{p}$ & $\frac{N m_{p} R^{2}}{3}$ \\
\hline
\end{tabular}

the responses between the $Z_{1}$ and $Z_{2}$ coordinates, $Z_{1}$ look "frozen" for $Z_{2}$ that is, the coordinates $Z_{1}$ in the subsystem (21) can be considered in their steady states. In turn, the response of the $Z_{2}$ coordinates is faster than the response of $\dot{\bar{\theta}}_{g}$ through (22), and so, the $Z_{2}$ coordinates can be considered in their steady states. The relation between the stability properties of the reduced system and the original system relies on the stability of the equilibria for all the subsystems involved and under the assumption $\varepsilon_{2}>\varepsilon_{1}>0$. In what follows, we proceed with the reduction process splitting it into two steps.

Step 1. Consider system (20) and (21) and $Z_{1}$ and $Z_{2}$ as the fast and the slow dynamics, respectively, so that $Z_{1}$ is at its 

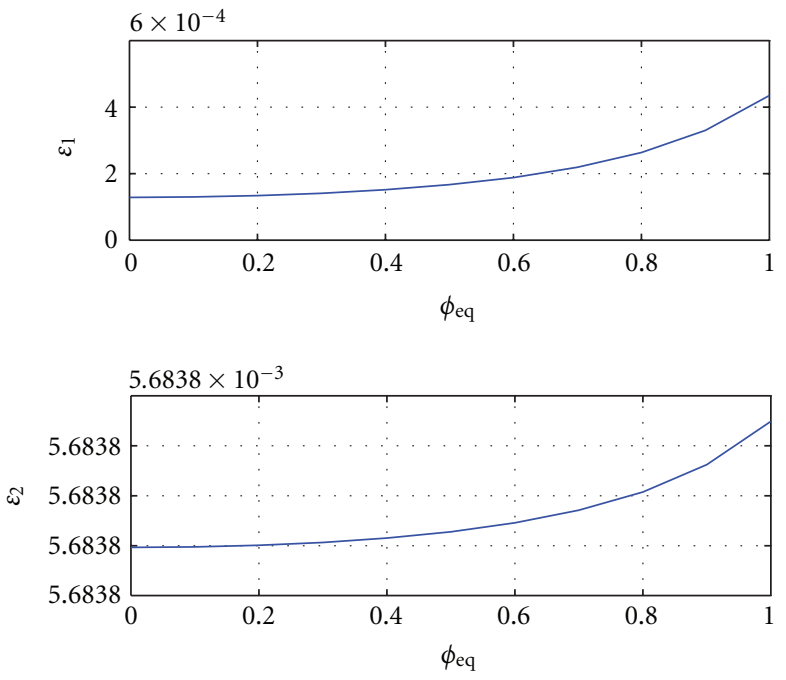

Figure 4: Parameters $\varepsilon_{1}$ and $\varepsilon_{2}$ as a function of $\phi_{\text {eq }}$.

quasisteady state $Z_{1}^{*}=\left[\bar{\theta}^{*}, \bar{\phi}^{*}, \dot{\bar{\theta}}^{*}, \dot{\bar{\phi}}^{*}\right]^{T}$. By setting $\varepsilon_{1}=0$, the equilibrium set becomes

$$
Z_{1}^{*}=\left[\begin{array}{c}
\beta_{1} \dot{\bar{\theta}}_{s}+\beta_{2} \dot{\bar{\theta}}_{g}+\beta_{3} \bar{F}_{T} \\
\alpha_{1} \dot{\bar{\theta}}_{s}+\alpha_{2} \dot{\bar{\theta}}_{g}+\alpha_{3} \bar{F}_{T} \\
0 \\
0
\end{array}\right],
$$

along with

$$
\begin{array}{ll}
\alpha_{1}=\frac{a_{5} b_{3}-b_{5} a_{3}}{a_{6} b_{5}-b_{6} a_{5}}, & \beta_{1}=\frac{a_{6} b_{3}-b_{6} a_{3}}{a_{5} b_{6}-b_{5} a_{6}}, \\
\alpha_{2}=\frac{a_{5} b_{4}-b_{5} a_{4}}{a_{6} b_{5}-b_{6} a_{5}}, & \beta_{2}=\frac{a_{6} b_{4}-b_{6} a_{4}}{a_{5} b_{6}-b_{5} a_{6}}, \\
\alpha_{3}=\frac{a_{5} b_{7}-b_{5} a_{7}}{a_{6} b_{5}-b_{6} a_{5}}, & \beta_{3}=\frac{a_{6} b_{7}-b_{6} a_{7}}{a_{5} b_{6}-b_{5} a_{6}} .
\end{array}
$$

By substituting (31) in (21), (21) becomes

$$
\varepsilon_{2} \frac{d Z_{2}}{d t}=A_{2} Z_{2}+w_{g_{2}} \dot{\bar{\theta}}_{g}+w_{r} \bar{T}_{r}+w_{t} \bar{T}_{g} .
$$

Step 2. Consider now the reduced system (33) and (22) and the fast and slow dynamics as $Z_{2}$ and $\dot{\bar{\theta}}_{g}$, respectively. Thus, by setting $\varepsilon_{2}=0$ the quasisteady state $Z_{2}^{*}=\left[\bar{\theta}_{s}^{*}, \dot{\bar{\theta}}_{s}^{*}\right]^{T}$ becomes

$$
Z_{2}^{*}=\left[\begin{array}{c}
-\frac{z_{3}}{z_{5}} \dot{\bar{\theta}}_{g}-\frac{z_{6}}{z_{5}} \bar{T}_{r}-\frac{z_{7}}{z_{5}} \bar{T}_{g} \\
0
\end{array}\right],
$$

and plugging (34) in (22), the reduced dynamics reads

$$
\frac{d \dot{\bar{\theta}_{g}}}{d t}=-\frac{B_{T}}{J_{T}} \dot{\bar{\theta}}_{g}+\frac{n_{g}}{J_{T}} \bar{T}_{r}-\frac{n_{g}}{J_{T}} \bar{T}_{g_{r}},
$$

where $\bar{T}_{g_{r}}$ is the generator torque referred to the rotor side, with $\bar{T}_{g_{r}}=n_{g} \bar{T}_{g}$,

$$
\begin{gathered}
B_{T}:=B_{r}+n_{g}^{2} B_{g}+B_{s}\left(1-\frac{1}{n_{g}}\right), \\
J_{T}:=J_{r}+n_{g}^{2} J_{g}+I_{p} \cos ^{2} \phi_{\mathrm{eq}} .
\end{gathered}
$$

Remark 4. We underscore here that the reduced equation (35) reproduces the dynamical structure commonly used for controlling wind turbines, where flexibility and torsion are neglected (see, e.g., $[7,8]$ ). In contrast, the previous analysis proves that taking into account the flexibility and the torsion, the same dynamical structure (35) arises with a suitable timescale separation. Additionally, as a contribution the timescale separation recovers the influence of the flexibility and torsion through (36) and (37). By comparing with $[7,8]$, the extra term $I_{p} \cos ^{2} \phi_{\mathrm{eq}}$ in (37) shows the influence of the flexibility of the blades.

Figure 5 shows the block structure according to (20)(22). The decoupled structure arises by zeroing the parameters $\varepsilon_{1}$ and $\varepsilon_{2}$.

Before concluding the section let us briefly comment on the stability properties of the singularly perturbed system (20)-(22). From our previous analysis it is clear that we cannot expect the states to converge to their quasisteady states unless some stability conditions are satisfied. The forthcoming analysis addresses those conditions. To this end, let us define a change of coordinates to shift the quasisteady states to the origin, which is given by

$$
\begin{gathered}
Y_{1}:=Z_{1}+A_{1}^{-1} h_{1}\left(Z_{2}, \dot{\bar{\theta}}_{g}, \overline{F_{T}}\right), \\
Y_{2}:=Z_{2}+A_{2}^{-1} h_{2}\left(Z_{1}, \dot{\bar{\theta}}_{g}, \bar{T}_{r}, \bar{T}_{g}\right),
\end{gathered}
$$

where

$$
\begin{gathered}
h_{1}:=w_{s_{1}} Z_{2}+w_{g_{1}} \dot{\bar{\theta}}_{g}+w_{f_{1}} \bar{F}_{T}, \\
h_{2}:=w_{s_{2}} Z_{1}+w_{g_{2}} \dot{\bar{\theta}}_{g}+w_{r} \bar{T}_{r}+w_{t} \bar{T}_{g},
\end{gathered}
$$

and, therefore, the system (20)-(22) in the new coordinates becomes

$$
\begin{gathered}
\varepsilon_{1} \frac{d Y_{1}}{d t}=A_{1} Y_{1}+\varepsilon_{1} \bar{h}_{1}, \\
\varepsilon_{2} \frac{d Y_{2}}{d t}=A_{2} Y_{2}+\varepsilon_{2} \bar{h}_{2}, \\
\frac{d \dot{\bar{\theta}}_{g}}{d t}=d_{2} \dot{\bar{\theta}}_{g}+w_{s_{3}}\left(Y_{2}-A_{2}^{-1} h_{2}\right)+d_{4} \bar{T}_{g},
\end{gathered}
$$

where $\bar{h}_{i}$ are the functions $h_{i}$ in the new coordinates, for $i=1,2$. The system (40)-(42) highlights the perturbed part of the system and, in turn, the time-scale separation. Thus, if the equilibria of $Y_{1}$ and $Y_{2}$ are asymptotically stable with $\epsilon_{1}=\epsilon_{2}=0$, then, it is reasonable to expect that 


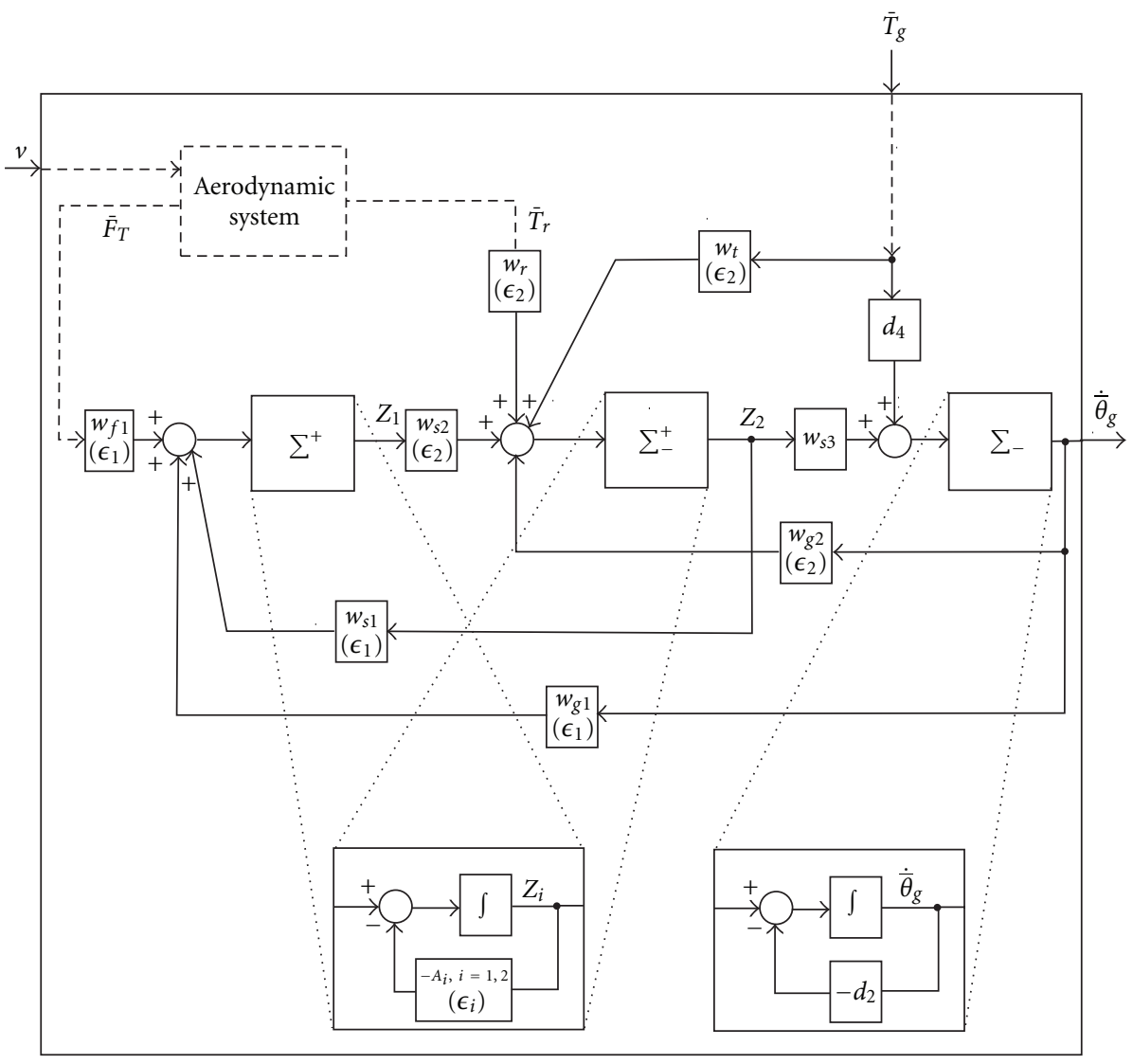

FIGURE 5: Block diagram of the wind turbine.

their respective solutions will reach an $\mathcal{O}\left(\varepsilon_{1}\right)$ and $\mathcal{O}\left(\varepsilon_{2}\right)$ neighbourhood of the origin during the boundary-layer transient. Therefore, the stability analysis of the whole system (40)-(42), or (20)-(22), is reduced to the stability analysis of the reduced system (35). Moreover, the existence of $\varepsilon_{1}$ and $\varepsilon_{2}$ such that this simplified analysis can be done is guaranteed if the matrices $A_{i}, i=1,2$, are Hurwitz and under some mild assumptions of smoothness. The following proposition states formally the utility of the approach.

Proposition 5. Consider the wind turbine system given by system (20)-(22). Suppose that the equilibrium of the reduced system (22) is exponentially stable with a specific control law on $\bar{T}_{g}$. Then, for any Hurwitz matrices $A_{i}, i=1,2$, there exist $\varepsilon_{2}^{*}>\varepsilon_{1}^{*}>0$ such that for $\varepsilon_{1}<\varepsilon_{1}^{*}$ and $\varepsilon_{2}<\varepsilon_{2}^{*}$ the equilibrium of the whole system (20)-(22) is exponentially stable.

Proof. The proof is based on the iterative application of Theorem 11.4 of [15].

\section{Observer Design for the Turbine Flexible Modes}

This section describes how the above developed wind turbine model and singular perturbation decomposition can be employed to estimate the system states and its potential for control applications.

Currently, most control algorithms depend on measurements from turbine structure and drive train for use in the control feedback [5]. Nonetheless, many advanced control techniques rely on the use of measurements that are not typically available for control. For example, most of the time wind turbines operate under the below-rated power conditions, on what is usually termed as region I of operation [1]. This regime of operation happens at wind speeds lower than the rated one, $V_{N}$, and is characterized in that the available wind power is lower than the maximum the turbine is designed to extract. Therefore, the usual generation objective in this region is maximizing power extraction preventing mechanical loads from exceeding certain bounds.

To maximize power extraction the turbine must be driven to operate on the maximum efficiency curve. The power extracted from the turbine can be expressed as

$$
P_{a}=\frac{1}{2} \rho \pi R^{2} C_{p}(\lambda, \beta) v^{3}
$$

where

$$
\lambda=\frac{\dot{\theta}_{r} R}{v}
$$


is the so-called tip speed ratio, $\dot{\theta}_{r}$ is the rotor speed, $v$ is the wind speed, $R$ is the rotor radius, $\beta$ is the blade pitch angle, and $\rho$ is the air density. Thus, the power extracted from the wind, $P_{a}$, is proportional to $v^{3}$, the surface of the aerodynamic disc, and the so-called power coefficient, $C_{p}$. The values of $C_{p}$ as a function of $\lambda$ and $\beta$ are usually numerically obtained using blade-element moment theory for every specific blade profile (see, e.g., the code WT-PERF [17] developed by NREL for more details on the subject).

The coefficient $C_{p}(\lambda, \beta)$ in (43) has a single maximum, $C_{p, \text { opt }}$, for specific optimal tip-speed ratio, $\lambda_{\text {opt }}$, and optimal pitch angle, $\beta_{\text {opt }}$, such that

$$
C_{p, \text { opt }}=C_{p}\left(\lambda_{\text {opt }}, \beta_{\text {opt }}\right) .
$$

A common strategy to maximize power extraction in region I consists in fixing the blade pitch angle to its optimal value, $\beta_{\text {opt }}$, while regulating the turbine speed to keep the tip-speed ratio as close as possible to $\lambda_{\text {opt }}$ manipulating the generator torque $T_{g}$. In other words, the control action, $T_{g}$, is designed to make the rotor speed tracks

$$
\dot{\theta}_{r, \mathrm{opt}}=\frac{\lambda_{\mathrm{opt}} v}{R} .
$$

It is worth noticing that $\dot{\theta}_{r \text {,opt }}$ depends only on the wind speed $v$ as $\lambda_{\text {opt }}$ is a constant and known value. Nonetheless, wind speed remains an unknown and fluctuating value that cannot be effectively measured in practice. The reason is that the wind exhibits in general a time-varying fullfield turbulent behaviour that cannot be summarized by a single magnitude on a specific point of the rotor disc. From this point of view, single anemometer measurements usually employed on top of turbine nacelle provides useful information for the trend of the average wind profile but cannot be efficiently used for control.

This problem is classically circumvented resorting to control techniques that do not require wind measurements for control. For instance, in [18] an indirect speed control strategy is formulated, based on the fact that a wind turbine is stable around any point of the optimal aerodynamic efficiency curve. With this idea, the controller is designed to track a constant, not estimated torque that approximates the optimal torque versus $\dot{\theta}_{r}$ curve. Adaptive control has also been employed in this context to design a controller that copes with unknown and not directly measurable wind speeds. Thus, in [19] a nonlinear adaptive control algorithm is devised such that the controller tracks an specified reference shaft speed in the presence of eventual model uncertainties.

Nonetheless, these methods exhibit low performance in presence of full-field turbulent winds, which has motivated the development of alternative methods to obtain indirect measurements of the wind, as in [20], where a Kalman filter is used to estimate the aerodynamic torque $\bar{T}_{r}$ and used as a reference for a linear torque tracking controller, or [8], where the aerodynamic torque estimated again with a Kalman filter is employed to infer an estimation of the effective wind speed impinging the rotor, and therefore used for control design.
This work proposes to extend the application of observers to also estimate the flexibility components of the wind turbine, that is, blades and tower deflection. These magnitudes are intimately related to the structural loads of the turbine and therefore to its lifespan. The problem of fatigue damage in wind turbines has been classically circumvented from the control point of view, using controllers tuned for smooth operation preventing the excitation of dangerous structural frequencies. This solution certainly leaves room for energy capture improvement, as the wind resources are not exploited to its fullest. The adoption of this solution is motivated, among other reasons, by the absence of measurements of the structure deformations, that could be employed for feedback in control.

4.1. Observer Design and Control Considerations. The timescale separation principle applied on the model of the wind turbine in (20)-(22) can be exploited to design an observer structure to estimate the flexible degrees of freedom of the model, namely $Z_{1}$.

Assuming the time-scale separation principle applies or, alternatively, that the drive train torsion can be discarded, an extended subsystem model can be built on the assumption that the rotor axial force, $\bar{F}_{T}$, is driven by a white noise of known variance. Note that $\bar{F}_{T}$ is the component of the wind over the turbine aerodynamic disc that mainly is responsible for the blades out of plane deflection and the fore-aft tower displacement, and as the aerodynamic torque, $\bar{T}_{r}$ cannot be directly measured. Under this assumption, $\bar{F}_{T}$ can be appended as an additional state of the plant, from (20), yielding

$$
\frac{d}{d t}\left[\begin{array}{c}
\varepsilon_{1} Z_{1} \\
\bar{F}_{T}
\end{array}\right]=\left[\begin{array}{cc}
A_{1} & \omega_{f_{1}} \\
0 & 0
\end{array}\right]\left[\begin{array}{c}
Z_{1} \\
\bar{F}_{T}
\end{array}\right]+\left[\begin{array}{c}
\omega_{g_{1}} \\
0
\end{array}\right] \dot{\bar{\theta}}_{g}+\left[\begin{array}{l}
0 \\
1
\end{array}\right] \xi .
$$

This structure could be in principle employed to build a standard Kalman filter to provide an estimate of the plant subspace $Z_{1}$ together with $\bar{F}_{T}$, provided an appropriate input is fed to the filter. In this case, and as remarkable difference with the estimators employed for the aerodynamic torque, $\bar{T}_{r}$, the filter cannot reconstruct the plant states just on the information of the rotor speed. This fact can be easily verified by checking the observability of system (47) together with system (22) with $\dot{\bar{\theta}}_{g}$ as an input.

From a physical point of view, this fact makes perfect sense, as we have checked that both systems, (20) and (22), can be uncoupled on a time-scale separation principle, yielding that subspace $Z_{1}$ is only marginally observable from input $\dot{\bar{\theta}}_{g}$. This fact makes necessary the introduction of an additional input to the system that provides enough information for the filter to reconstruct the states of (47), without the need of implementing additional sophisticated and/or expensive measurement devices on the turbine. Careful consideration of the possibilities suggested the introduction of an accelerometer on top of the turbine nacelle, which provided fore-aft tower acceleration measurement. Current state-of-the-art solid state accelerometers are cheap yet effective and reliable for the task at hand. This additional 
measurement can be easily incorporated to system (47) considering an additional equation as

$$
y=H \cdot\left[\begin{array}{lllll}
a_{5} & a_{6} & a_{1} & a_{2} & a_{7}
\end{array}\right]\left[\begin{array}{c}
Z_{1} \\
\bar{F}_{T}
\end{array}\right],
$$

where parameters are defined as in equation (17) and $H$ represents the tower height. In other words, the nacelle foreaft acceleration is approximated in the model as $H \cdot \ddot{\bar{\theta}}$.

Remark 6. Despite this additional input, it can be checked that the overall system observability condition (checking for instance that the observability matrix of the system is full rank [21]) for system (47) and (48) fails. It is interesting, nonetheless, to analyze the structure of the observable and unobservable subspaces of the system, a task that can be performed resorting to standard linear control techniques [21]. Thus, a closer look at the problem reveals that $\bar{F}_{T}$ is the only state not observable from the newly introduced input, while $Z_{1}$ can be reconstructed. This allows us to build a Kalman filter on the observable subspace of (47) where state $\bar{F}_{T}$ is removed.

3.1. Reduced Dynamics. This section provides some results on the performance of the flexible-modes filter described above. Simulations were performed with the fatigue, aerodynamics, structures and turbulence (FAST) code developed by NREL. It is an aeroelastic wind turbine simulator that uses an assumed mode method to model flexible blades and tower components. A multibody model is employed for other components.

In these simulations the AWT, three-bladed WP 1.5 MW wind turbine provided by NREL was selected. This turbine features a hub height of $42 \mathrm{~m}$, with $33.25 \mathrm{~m}$ length blades and variable speed-variable pith control capabilities.

In order to reflect realistic operation conditions, the study has been performed considering a 16DOF model, taking into account flexibility associated with torsional motion between the generator and hub/rotor together with fore-aft tower flexibility and out-of-plane blade flapping. Experiments have been developed for full-field turbulent wind profile with $12 \mathrm{~m} / \mathrm{s}$ average speed, while a simple PI control has been applied on the control part to make the turbine operate at constant $5 \mathrm{rad} / \mathrm{s}$ despite wind fluctuations.

Figures 6 and 7 show, respectively, the estimated and measured (from simulation) deflection of tower and blades. For the blades, FAST simulator provides three distinct values, one for each blade, so an average of these values has been taken to be compared to the single blade deflection DOF available from the estimator model. It can be observed that the estimated values for the tower deflection are quite accurate in general, collecting the main dynamics of the system both in amplitude and average frequency. Tracking of the blade deflection is nonetheless more inaccurate, though the main trend is correctly reproduced. These results should not surprise us, as the information employed to reconstruct the flexible states, the nacelle fore-aft acceleration (see Figure 8), is directly linked to the tower DOF. The blade

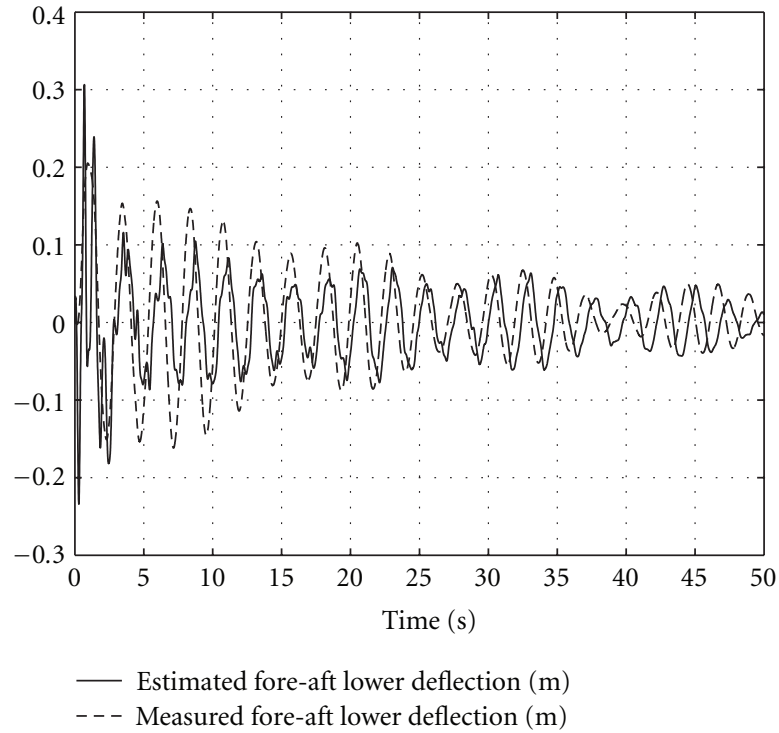

Figure 6: Estimated fore-aft tower deflection versus measured (simulated) fore-aft tower deflection.

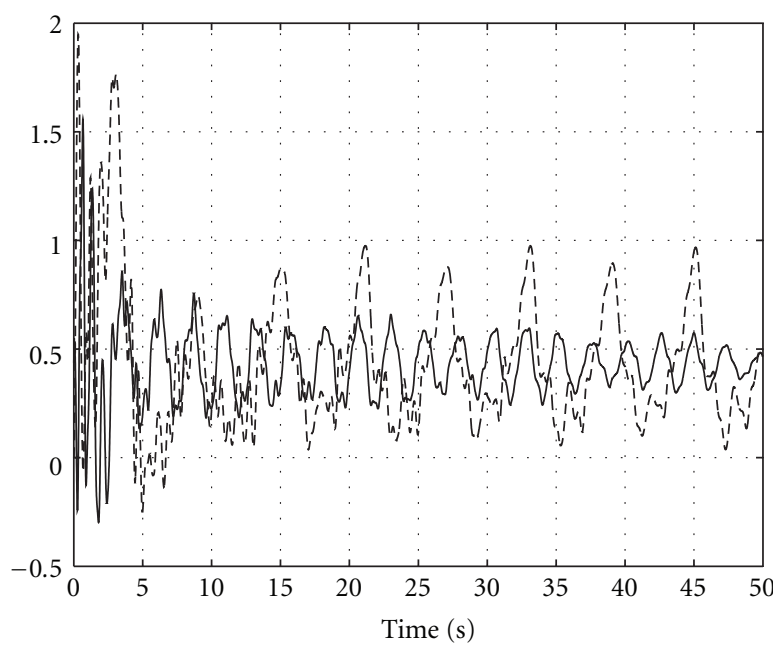

- Estimated out-of-plane average blade deflection (m)
- - - Measured out-of-plane average blade deflection $(\mathrm{m})$

FIGURE 7: Estimated out-of-plane averaged blade deflection versus measured (simulated) out-of-plane averaged blade deflection.

deflection DOF is much more difficult to estimate first because the linear model employed to build the filter fails to collect all dynamical effects present in the aerodynamic disc and second because the nacelle fore-aft acceleration is only indirectly linked to the behaviour of the blades.

Nonetheless, it is worth to mention that for general control purposes, perfect tracking of these signals is not required. Relevant information about load effects on the structure can be extracted from the main frequencies of oscillation together with their maximum amplitudes, and these data are reasonably well approximated by the estimation structure proposed. 
TABle 5: Parameters for the model (20)-(22).

\begin{tabular}{|c|c|c|c|}
\hline Parameters & Value & Parameters & Value \\
\hline $\bar{a}_{1}$ & $-\frac{m_{3} \bar{c}_{11}}{m_{1} m_{4} n_{g}^{2}}$ & $\bar{b}_{1}$ & $\frac{m_{2} \bar{c}_{11}}{m_{1} m_{4} n_{g}^{2}}$ \\
\hline $\bar{a}_{2}$ & $\frac{m_{2} \bar{c}_{22}}{m_{1} m_{4} n_{g}^{2}}$ & $\bar{b}_{2}$ & $-\frac{\bar{c}_{22}}{m_{4} n_{g}^{2}}$ \\
\hline $\bar{a}_{3}$ & $\frac{m_{2} \bar{c}_{23}}{m_{1} m_{4} n_{g}^{2}}$ & $\bar{b}_{3}$ & $-\frac{\bar{c}_{23}}{m_{4} n_{g}^{2}}$ \\
\hline $\bar{a}_{4}$ & $\frac{m_{2} \bar{c}_{23}}{m_{1} m_{4} n_{g}^{3}}$ & $\bar{b}_{4}$ & $-\frac{\bar{c}_{23}}{m_{4} n_{g}^{3}}$ \\
\hline $\bar{a}_{5}$ & $-\frac{m_{3} k_{1}}{m_{1} m_{4} n_{g}^{2}}$ & $\bar{b}_{5}$ & $\frac{m_{2} k_{1}}{m_{1} m_{4} n_{g}^{2}}$ \\
\hline $\bar{a}_{6}$ & $\frac{m_{2} k_{3}}{m_{1} m_{4} n_{g}^{2}}$ & $\bar{b}_{6}$ & $-\frac{k_{3}}{m_{4} n_{g}^{2}}$ \\
\hline $\bar{a}_{7}$ & $\frac{N\left(m_{3} H \cos \theta_{\mathrm{eq}}-m_{2} r_{b} \cos \phi_{\mathrm{eq}}\right)}{m_{1} m_{4} n_{g}^{2}}$ & $\bar{z}_{1}$ & $\frac{\bar{c}_{23} d_{1}}{m_{4} z_{3}}$ \\
\hline $\bar{b}_{7}$ & $-\frac{N\left(m_{2} H \cos \theta_{\mathrm{eq}}-m_{1} r_{b} \cos \phi_{\mathrm{eq}}\right)}{m_{1} m_{4} n_{g}^{2}}$ & $\bar{z}_{2}$ & $\frac{d_{1} z_{2}}{z_{3}}$ \\
\hline $\bar{z}_{6}$ & $\frac{d_{1}}{m_{4} z_{3}}$ & $\bar{z}_{3}$ & $d_{1}$ \\
\hline $\bar{z}_{7}$ & $-\frac{d_{1} d_{4}}{z_{3} n_{g}}$ & $\bar{z}_{5}$ & $\frac{d_{1} z_{5}}{z_{3}}$ \\
\hline
\end{tabular}

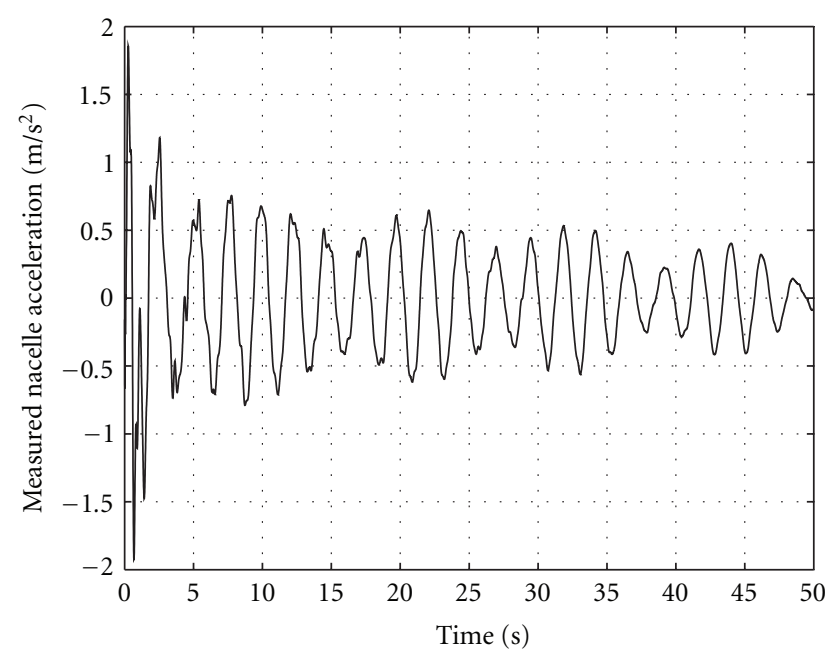

Figure 8: Measurement of the accelerometer on the nacelle (simulated).

\section{Conclusion}

In this work, a model for wind turbines taking into account the flexibility and torsional forces has been developed. The common and worth assumption of a reduced mechanical model of order one is justified through a time-scale separation based on the singularly perturbed structure of the system. The identification of the key parameters for the decoupling and some new insights for the design and control of the wind turbine are given. Moreover, the singularly perturbed structure together with the fore-aft acceleration in the the nacelle as an available output allows us to design a new estimator for the whole flexible modes of the wind turbine, that is, tower and blades.

\section{Appendices}

\section{A. Parameters for Model (17) -(19)}

The corresponding parameters are given in Table 4 .

\section{B. Definitions and Parameters for the Model (20)-(22)}

The corresponding matrices of the equations (20)-(22) have been defined as

$$
\Sigma_{Z_{1}}: A_{1}=\left[\begin{array}{cccc}
0 & 0 & 1 & 0 \\
0 & 0 & 0 & 1 \\
\bar{a}_{5} & \bar{a}_{6} & \bar{a}_{1} & \bar{a}_{2} \\
\bar{b}_{5} & \bar{b}_{6} & \bar{b}_{1} & \bar{b}_{2}
\end{array}\right], \quad w_{s_{1}}=\left[\begin{array}{cc}
0 & 0 \\
0 & 0 \\
0 & \bar{a}_{3} \\
0 & \bar{b}_{3}
\end{array}\right],
$$




$$
\begin{aligned}
w_{g_{1}}=\left[\begin{array}{c}
0 \\
0 \\
\bar{a}_{4} \\
\bar{b}_{4}
\end{array}\right], \quad w_{f_{1}}=\left[\begin{array}{c}
0 \\
0 \\
\bar{a}_{7} \\
b_{7}
\end{array}\right], \\
\Sigma_{Z_{2}: A_{2}}=\left[\begin{array}{cc}
0 & 1 \\
\bar{z}_{5} & \bar{z}_{2}
\end{array}\right], \quad w_{s_{2}}=\left[\begin{array}{llll}
0 & 0 & 0 & 0 \\
0 & 0 & 0 & \bar{z}_{1}
\end{array}\right], \\
w_{g_{2}}=\left[\begin{array}{c}
0 \\
\bar{z}_{3}
\end{array}\right], \quad w_{r}=\left[\begin{array}{c}
0 \\
\bar{z}_{6}
\end{array}\right], \quad w_{t}=\left[\begin{array}{c}
0 \\
\bar{z}_{7}
\end{array}\right], \\
\Sigma_{\overline{\bar{\theta}}_{g}}: w_{s_{3}}=\left[\begin{array}{c}
d_{3} \\
d_{1}
\end{array}\right] .
\end{aligned}
$$

and its corresponding parameters are given in Table 5.

\section{Acknowledgment}

The authors would like to acknowledge MAEC-AECID (Ministerio de Asuntos Exteriores y de CooperaciónAgencia Española de Cooperación Internacional para el Desarrollo) for funding this work.

\section{References}

[1] F. D. Bianchi, H. de Battista, and R. J. Mantz, Wind Turbine Control Systems: Principles, Modelling and Gain Scheduling Design, Springer, Heidelberg, Germany, 2007.

[2] B. Boukhezzar and H. Siguerdidjane, Comparison Between Linear and Nonlinear Control, Strategies for Variable Speed Wind Turbine Power Capture Optimization, Ecologic Vehicles, Renewable Energies, Monaco, 2009.

[3] M. M. Hand, "Variable-speed wind turbine controller systematic design methodology: a comparison of non-linear and linear model-based designs," NREL Report number TP-50025540, National Renewable Energy Laboratory, Golden, Colo, USA, 1999.

[4] K. A. Stol, "Disturbance tracking control and blade load mitigation for variable-speed wind turbines," Journal of Solar Energy Engineering, Transactions of the ASME, vol. 125, no. 4, pp. 396-401, 2003.

[5] J. H. Laks, L. Y. Pao, and A. D. Wright, "Control of wind turbines: past, present, and future," in Proceedings of the American Control Conference (ACC '09), pp. 2096-2103, St. Louis, Mo, USA, June 2009.

[6] A. Khamlichi, B. Ayyat, R. O. B. Zarouala, and C. V. Venegas, "Advanced control based on extended Kalman filter for variable speed wind turbine," Australian Journal of Basic and Applied Sciences, vol. 5, no. 9, pp. 636-644, 2011.

[7] B. Boukhezzar, H. Siguerdidjane, and M. Maureen Hand, "Nonlinear control of variable-speed wind turbines for generator torque limiting and power optimization," ASME Journal of Solar Energy Engineering, vol. 128, no. 4, pp. 516-530, 2006.

[8] B. Boukhezzar, L. Lupu, H. Siguerdidjane, and M. Hand, "Multivariable control strategy for variable speed, variable pitch wind turbines," Renewable Energy, vol. 32, no. 8, pp. 1273-1287, 2007.
[9] P. Novak, I. Jovik, and B. Schmidtbauer, "Modeling and identification of drive-system dynamics in a variable-speed wind turbine," in Proceedings of the IEEE Conference on Control Applications, vol. 1, pp. 233-238, August 1994.

[10] B. Beltran, T. Ahmed-Ali, and M. E. H. Benbouzid, "Sliding mode power control of variable-speed wind energy conversion systems," IEEE Transactions on Energy Conversion, vol. 23, no. 2, pp. 551-558, 2008.

[11] A. A. Shabana, Dynamics of Multibody Systems, Cambridge, Mass, USA, 2005.

[12] J. Jonkman, S. Butterfield, W. Musial, and G. Scott, "Definition of a 5-MW reference wind turbine for offshore system development," Tech. Rep. NREL/TP-500-38060, 2009.

[13] J. Jonkman and M. Buhl Jr., "FAST user's guide," Tech. Rep. NREL/EL-500-38230, 2005.

[14] P. V. Kokotovic, J. J. Allemong, J. R. Winkelman, and J. H. Chow, "Singular perturbation and iterative separation of time scales," Automatica, vol. 16, no. 1, pp. 23-33, 1980.

[15] H. K. Khalil, Nonlinear Systems, Prentice-Hall, 3rd edition, 2000.

[16] J. A. Acosta and M. López-Martínez, "A nonlinear strategy to control unstable underactuated mechanical systems with underactuation $\geq 1$. Applications to control augmentations," Open Automation and Control Systems Journal, vol. 2, no. 1, pp. 13-20, 2009.

[17] M.L. Buhl, "WT PERF user's guide," Tech. Rep., National Renewable Energy Laboratory, Golden, Colo, USA, 2004.

[18] W. E. Leithead and B. Connor, "Control of variable speed wind turbines: design task," International Journal of Control, vol. 73, no. 13, pp. 1189-1212, 2000.

[19] Y. D. Song, B. Dhinakaran, and X. Y. Bao, "Variable speed control of wind turbines using nonlinear and adaptive algorithms," Journal of Wind Engineering and Industrial Aerodynamics, vol. 85, no. 3, pp. 293-308, 2000.

[20] H. Vihriala, R. Peralaand, P. Makila, and L. Soderlund, "A gearless wind power drive: part 2: performance of control system," in Proceedings of the European Wind Energy Conference, 2001.

[21] F. W. Fairman, Linear Control Theory: The State Space Approach, John Wiley \& Sons, 1998. 

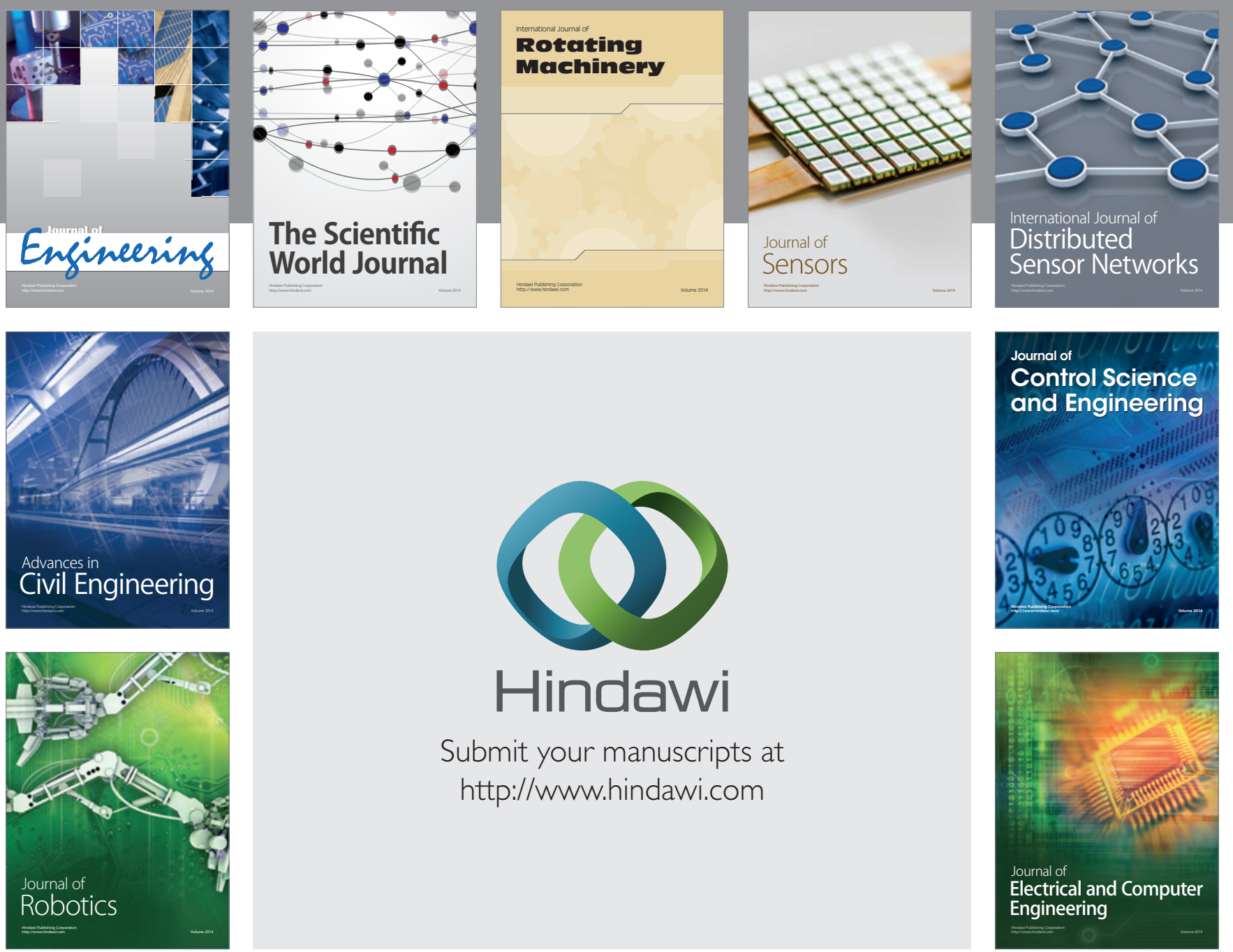

Submit your manuscripts at

http://www.hindawi.com
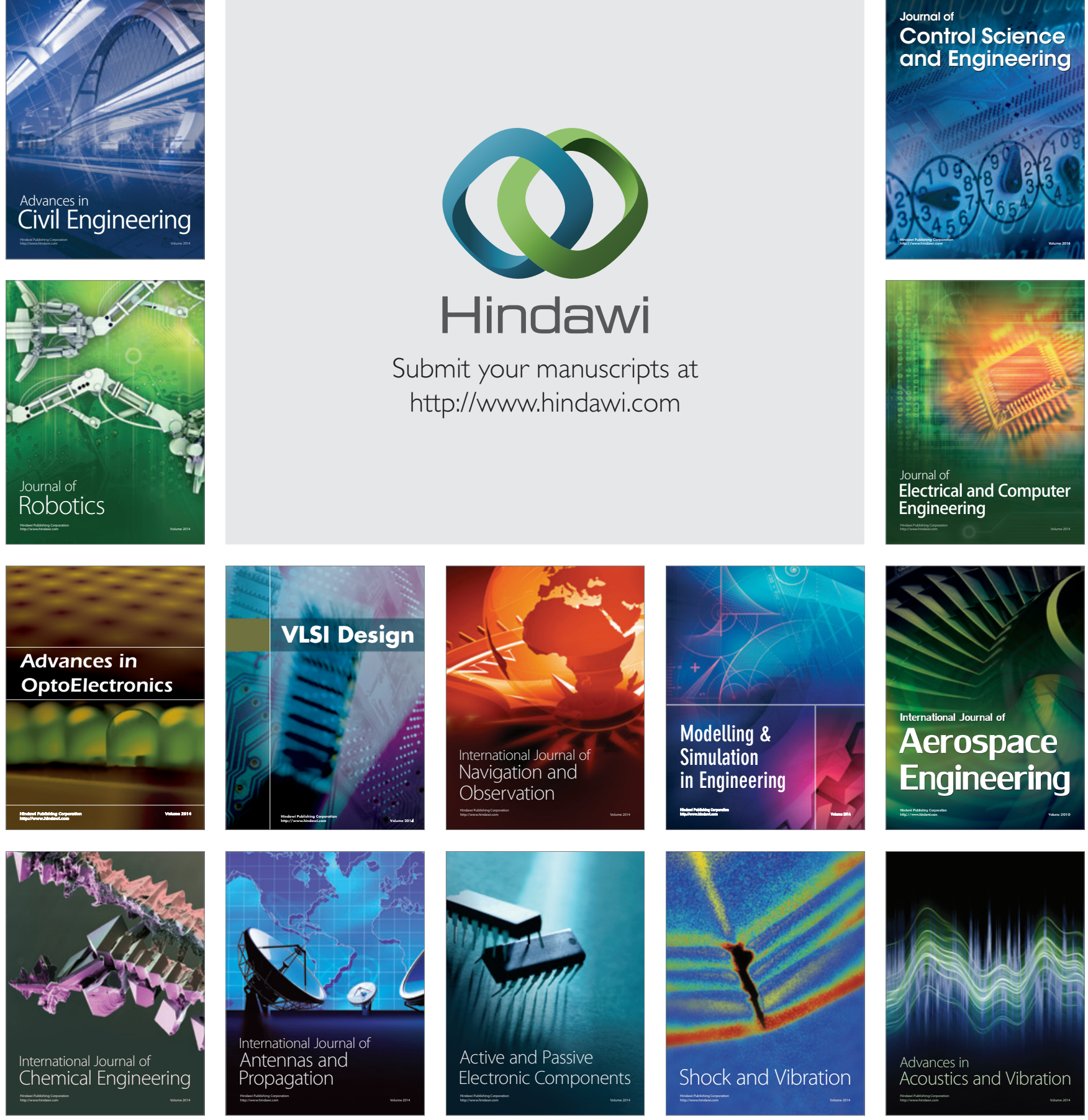